\title{
Integrated tunneling sensor for nanoelectromechanical systems
}

\author{
Sadewasser, S.; Abadal, G.; Barniol, N.; Dohn, Søren; Boisen, Anja
}

Published in:

Applied Physics Letters

Link to article, DOI:

$10.1063 / 1.2362593$

Publication date:

2006

Document Version

Publisher's PDF, also known as Version of record

Link back to DTU Orbit

Citation (APA):

Sadewasser, S., Abadal, G., Barniol, N., Dohn, S., \& Boisen, A. (2006). Integrated tunneling sensor for nanoelectromechanical systems. Applied Physics Letters, 89(17), 173101. https://doi.org/10.1063/1.2362593

\section{General rights}

Copyright and moral rights for the publications made accessible in the public portal are retained by the authors and/or other copyright owners and it is a condition of accessing publications that users recognise and abide by the legal requirements associated with these rights.

- Users may download and print one copy of any publication from the public portal for the purpose of private study or research.

- You may not further distribute the material or use it for any profit-making activity or commercial gain

- You may freely distribute the URL identifying the publication in the public portal

If you believe that this document breaches copyright please contact us providing details, and we will remove access to the work immediately and investigate your claim. 


\title{
Integrated tunneling sensor for nanoelectromechanical systems
}

\author{
S. Sadewasser ${ }^{\text {a) }}$ \\ Instituto de Microelectrónica de Barcelona, CNM-CSIC, Campus UAB, 08193 Bellaterra, Spain \\ G. Abadal and N. Barniol \\ Departament de Enginyeria Electrònica, Universitat Autònoma de Barcelona, 08193 Bellaterra, Spain
}

S. Dohn and A. Boisen

MIC-Department of Micro and Nanotechnology, NanoDTU, Technical University of Denmark, Building 345, 2800 Lyngby, Denmark

L. Fonseca and J. Estéve ${ }^{\text {b) }}$

Instituto de Microelectrónica de Barcelona, CNM-CSIC, Campus UAB, 08193 Bellaterra, Spain

(Received 2 March 2006; accepted 1 September 2006; published online 23 October 2006)

Transducers based on quantum mechanical tunneling provide an extremely sensitive sensor principle, especially for nanoelectromechanical systems. For proper operation a gap between the electrodes of below $1 \mathrm{~nm}$ is essential, requiring the use of structures with a mobile electrode. At such small distances, attractive van der Waals and capillary forces become sizable, possibly resulting in snap-in of the electrodes. The authors present a comprehensive analysis and evaluation of the interplay between the involved forces and identify requirements for the design of tunneling sensors. Based on this analysis, a tunneling sensor is fabricated by Si micromachining technology and its proper operation is demonstrated. () 2006 American Institute of Physics.

[DOI: $10.1063 / 1.2362593$ ]

In recent years nanoelectromechanical systems (NEMSs) have received steadily increasing attention, motivated by numerous applications envisaged and their possibilities in future technologies. ${ }^{1}$ One of the interesting properties of NEMSs is their high frequency of resonance; this makes them attractive for sensing and systems applications, for example, in highly sensitive mass detection ${ }^{2}$ and radio frequency signal processing. ${ }^{3}$ One of the current challenges is the problem of detection, namely, the sensor, which connects the NEMSs to the surrounding electrical circuitry. Various sensor concepts have been developed for microelectromechanical systems (MEMSs), the most popular relying on capacitive detection. However, when decreasing the length scale from micrometers to nanometers, capacitive detection becomes increasingly difficult, due to the reduction of the active capacitance compared to the parasitic ones. An amplifier located very close to the NEMS based transducer could help in reducing the effect of stray capacitance components; ${ }^{4}$ however, this approach is usually limited by the complementary metal oxide semiconductor technology constraints. Therefore, alternative sensor concepts have been proposed for NEMSs, ${ }^{1}$ of which a sensor based on the electron tunneling effect is especially attractive.

This type of sensor relies on the tunneling quantum mechanical effect. When the gap between two conductors is small enough, an electron can tunnel through the energy barrier from one side to the other with a finite probability. The tunneling current is an exponential function of the distance $d$ between the electrodes and the tunneling barrier $\Phi$, which corresponds to the effective work function of the electrodes:

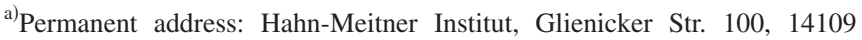
Berlin, Germany.

${ }^{b)}$ Electronic mail: jaume.esteve@cnm.es
}

$$
I=I_{0} \exp \left(-\frac{2}{\hbar} \sqrt{2 m \Phi} d\right)
$$

where $I_{0}$ depends on the applied voltage across the gap and $m$ is the electron mass. Practically, this effect is relevant for distances on the order of $1 \mathrm{~nm}$ and below. The tunneling current does not depend on the dimensions of the electrode (as in the case of the capacitive detection), which makes it especially well suited for NEMS applications. The tunneling current between a sharp tip and a sample is used in the scanning tunneling microscope (STM) to image topography. ${ }^{5}$

Only a few developments of tunneling sensors have been reported. Motivated by the extreme sensitivity of electron tunneling, Kenny et al. ${ }^{6}$ developed a tunnel sensor for motion detection using Si bulk micromachining. A vertical tip and the electrode were fabricated on individual chips which were subsequently bonded together. The electrode was moved by electrostatic actuators to approach it to the tip. This sensor was applied in an infrared detector ${ }^{7}$ and an accelerometer with micro- $g$ sensitivity. ${ }^{8}$

In this letter we present a detailed investigation of the physical forces involved in the operation of a lateral tunneling sensor. Based on this analysis we present the design and single-mask fabrication of this sensor on a single chip using Si micromachining technology and show its operation.

The motivation of the presented study is to develop a lateral tunneling sensor for the detection of motion in a NEMS device (which will be represented as a fixed electrode in its realization presented below). A significant difficulty in the manufacturing of a lateral tunneling sensor is the fabrication of a small gap between the sensor and the NEMS, which should be on the order of $1 \mathrm{~nm}$. Current nanotechnological fabrication techniques reach a lower limit of $\sim 10 \mathrm{~nm}$. The concept of the presented tunneling sensor is based on an adjustable gap between the sensor and the NEMS. For this purpose the sensor is composed of a tip which can be translated with respect to the NEMS. The 

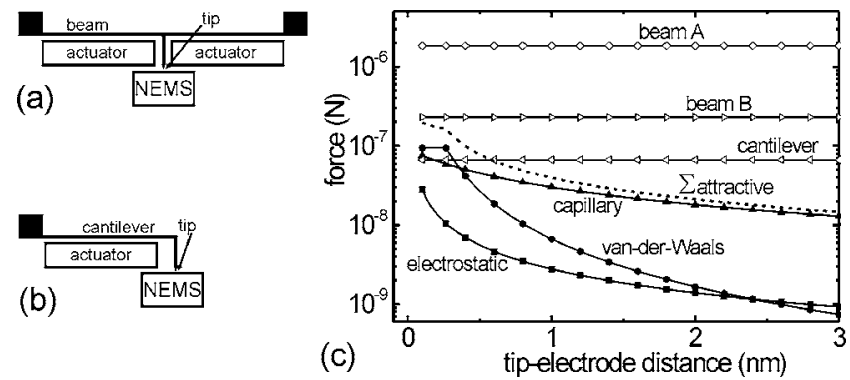

(b)

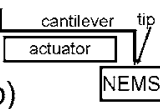

(c)

tipelectrode distance $(\mathrm{nm})$

FIG. 1. Design of two tunneling sensor structures that allow approaching a tip to a NEMS by means of (a) a doubly clamped beam and (b) a cantilever. (c) Comparison of the attractive electrostatic (solid squares), van der Waals (solid circles), and capillary forces (solid triangles) and their sum (dashed line) with the restoring mechanical force of different sensor structures (open symbols). See text for details.

structure for moving the tip is a MEMS consisting of a cantilever or a doubly clamped beam which is flexible in the plane of the substrate. This movable structure carries a tip which can be approached to the NEMS. The design principle is schematically shown in Fig. 1. Electrostatic actuators produce a bending of a beam [Fig. 1(a)] or a cantilever [Fig. 1(b)]. Applying the appropriate voltage to the actuator(s) the tip-NEMS gap is reduced to allow for electron tunneling. However, when the tip is in close proximity, the forces between tip and NEMS can result in a snap-in, thus bringing both in physical contact. This effect is well known from electrostatic actuators, where the electrostatic force resulting from the increasing voltage applied between the electrodes becomes larger than the restoring mechanical force of the bend beam/cantilever; this occurs when the actuator gap is reduced to $\sim 44 \%$ of the initial gap. ${ }^{9}$ In the present case a tip in front of an electrode has to be considered; for the small distances of interest here, the electrostatic force $F_{\mathrm{el}}$ is assumed to correspond to the geometry of a sphere in front of a plane: ${ }^{10}$

$$
F_{\mathrm{el}}=-\frac{\pi \varepsilon_{0} R}{d} V_{t}^{2}
$$

where $V_{t}$ is the tunneling voltage (which is much smaller than the actuator voltage), $R$ is the tip radius, and $d$ is the tip-electrode distance.

The restoring force is given by the mechanical properties of the beam/cantilever: ${ }^{11}$

$$
F_{\text {mech }}=A \frac{E t w^{3}}{l^{3}} x
$$

where $E=180 \times 10^{9} \mathrm{~N} \mathrm{~m}^{-2}$ is Young's modulus for silicon, $x$ the displacement from the equilibrium position and $t$ the thickness, $w$ the width (in direction of the motion), and $l$ the length of the beam. A is a geometric constant: $A=1 / 4$ for a cantilever and $A=32$ for a doubly clamped beam.

In the present case, where a sufficiently small distance for tunneling is required, additional forces have to be considered; namely, the van der Waals force and capillary forces become relevant. The van der Waals force can be approximated by using the model of a sphere in front of a plane: ${ }^{12}$

$$
F_{\mathrm{vdW}}=-\frac{H R}{6 d^{2}},
$$

where $H$ is the Hamaker constant. At distances below an intermolecular distance $a_{0}, F_{\mathrm{ydW}}$ is replaced by the adhesion

Downloaded 17 Jun 2010 to 192.38.67.112. Redistribution subject

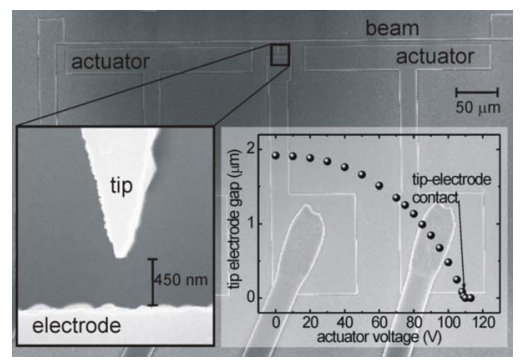

FIG. 2. Scanning electron microscopy image of a tunneling sensor. The beam is moved by electrostatic actuators approaching the tip to an opposing electrode. The left inset shows a zoom into the tip-electrode region (actuator voltage $=100 \mathrm{~V}$ ) and the right inset shows the tip-electrode gap as a function of the applied actuator voltage.

force $F_{\text {adh }}=-4 \pi R \gamma_{\mathrm{H}_{2} \mathrm{O}}$ to avoid divergence $\left(\gamma_{\mathrm{H}_{2} \mathrm{O}}\right.$ $=0.072 \mathrm{~J} \mathrm{~m}^{-2}$ is the liquid vapor interfacial energy). ${ }^{13}$ For sensor operation under ambient condition, the capillary force has to be considered; a meniscus forms spontaneously, when the distance becomes smaller than twice the Kelvin radius: ${ }^{14}$

$$
\kappa=-\frac{\gamma_{\mathrm{H}_{2} \mathrm{O}}}{n k T \ln \left(p / p_{\mathrm{sat}}\right)},
$$

where $n$ is the number density of water and $p / p_{\text {sat }}$ is the relative humidity ( $\kappa \approx 1.5 \mathrm{~nm}$ at $70 \%$ relative humidity). The capillary force has been described by Zitzler et al.: ${ }^{13}$

$$
F_{\text {cap }}=-\frac{4 \pi \gamma_{\mathrm{H}_{2} \mathrm{O}} R}{1+d / \tau},
$$

where $\tau$ is the thickness of the water film, which depends on the humidity. ${ }^{13}$

For proper operation of a tunneling sensor, the restoring mechanical force of the tip supporting structure has to be larger than the sum of all interaction forces $F_{\text {int }}$, down to distances $d$ sufficiently small for tunneling to occur, i.e., $d<1 \mathrm{~nm}$ :

$$
F_{\text {mech }}>\left|F_{\text {int }}\right|=\left|F_{\text {el }}+F_{\text {vdW }}+F_{\text {cap }}\right| \text {. }
$$

Figure 1(c) gives an overview of the distance dependence of the various forces. The mechanical force is considered for three different arrangements: (i) a cantilever of length $l$ $=300 \mu \mathrm{m}$ and width $w=2 \mu \mathrm{m}$ (open triangles, left), (ii) a doubly clamped beam of $l=500 \mu \mathrm{m}$ and $w=2 \mu \mathrm{m}$ (beam A, open diamonds), and (iii) a doubly clamped beam of same length and $w=1 \mu \mathrm{m}$ (beam $\mathrm{B}$, open triangles, right). All structures have a thickness of $5 \mu \mathrm{m} .{ }^{15}$ The parameters used for the various forces are $H=40 \times 10^{20} \mathrm{~J}, \quad V_{t}=1 \mathrm{~V}$, $h=10 \mu \mathrm{m}, R=100 \mathrm{~nm}$, and $\tau=0.5 \mathrm{~nm}$. In the case of dis-

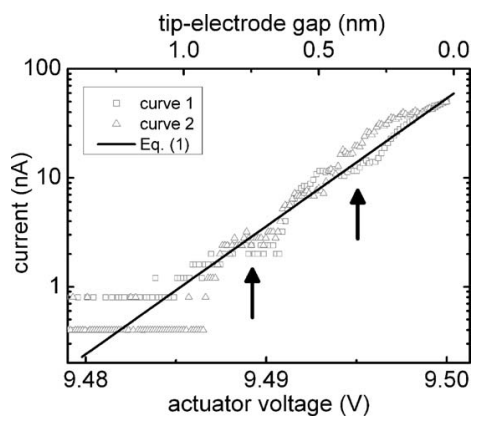

FIG. 3. Tip-electrode current upon approaching the tip by applying a voltage to the electrostatic actuators. A tunneling voltage of $-1 \mathrm{~V}$ is applied to the electrode. The solid line is a fit using the tunneling equation [Eq. (1)]. 


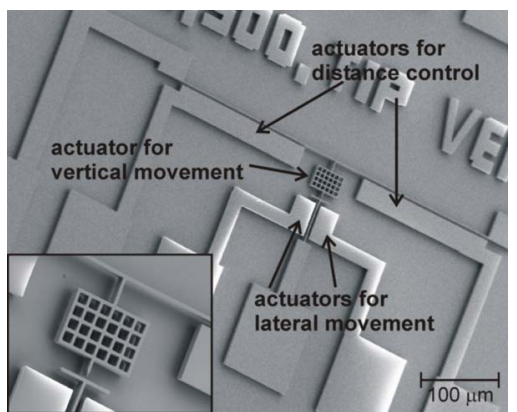

FIG. 4. Integrated STM consisting of a doubly clamped beam carrying a tip. Electrostatic actuators provide the possibility for lateral electrode (sample) motion, vertical tip motion, and for the tip approach to a counter electrode. The inset shows the central part of the structure in more detail.

tances $d>1 \mathrm{~nm}$ the mechanical restoring force is larger than $F_{\text {int }}$ for all three mechanical structures. For the cantilever the snap-in distance, below which $F_{\text {mech }}<F_{\text {int }}$, is at $d=0.6 \mathrm{~nm}$. For beam B at distances below $0.3 \mathrm{~nm} F_{\text {int }}$ reaches the same order of magnitude as the restoring force. Therefore, beam A, where $F_{\text {mech }} \gg\left|F_{\text {int }}\right|$ for all $d$, is the most suitable arrangement for a tunneling sensor based on a movable tip. ${ }^{16}$

Tunneling sensors based on the design of beam A were fabricated using silicon micromachining processes. A silicon on insulator wafer consisting of $5 \mu \mathrm{m} \mathrm{Si}$ on $2 \mu \mathrm{m} \mathrm{SiO}{ }_{2}$ was provided with regular photoresist to define the geometry. An anisotropic dry process (Alcatel A601) was used to vertically etch the complete silicon layer down to the buried $\mathrm{SiO}_{2}$ layer. Then, HF vapors are used to laterally underetch $\sim 3 \mu \mathrm{m}$ of this oxide, which liberates all structures of width less than $6 \mu \mathrm{m}$. The use of HF vapors instead of a liquid guarantees that the structures do not stick to the substrate due to drying water droplets. Subsequently, the structure is sputter coated with $\mathrm{Ti}(30 \mathrm{~nm}), \mathrm{Ni}(50 \mathrm{~nm})$, and $\mathrm{Au}(100 \mathrm{~nm})$ to ensure a conductive surface for proper tunneling conditions. For testing purposes, the tunneling structures contain a fixed electrode representing the NEMS. A scanning electron microscopy image of the tunneling sensor is presented in Fig. 2. The beam, the actuators, and the electrode are contacted via contact pads. The initial actuator gap of this structure is $\sim 1.9 \mu \mathrm{m}$. The left inset shows a zoom into the region of the tip and electrode with an applied actuator voltage of $100 \mathrm{~V}$. The right inset shows the tip-electrode gap as a function of applied actuator voltage (the beam is grounded) as measured in a scanning electron microscope; tip-electrode contact is achieved at $\sim 110 \mathrm{~V}$.

For the tunneling experiments a similar structure but with a smaller initial tip-electrode distance was used. A tunneling voltage of $V_{\text {electrode }}=-1 \mathrm{~V}$ was applied to the electrode, with the tip and the beam grounded. The measurement of the tunneling current was obtained by applying a fixed dc voltage of $V_{\mathrm{dc}}=46 \mathrm{~V}$ for coarse approach and an additional ac voltage of $V_{\mathrm{ac}}=9.54 \mathrm{~V}$ (peak to peak) using a frequency of $10^{-3} \mathrm{~Hz}$. An oscilloscope was used to read out the currents supplied by a current amplifier (SRS 570) connected to the electrode. Two typical curves for the tunneling current as a function of the applied ac voltage are shown in Fig. 3. The motion of the structure was observed in an optical microscope simultaneous to the tunneling measurement. From this observation the tip movement in the relevant range is determined to be $67 \pm 7 \mathrm{~nm} / \mathrm{V}$ (upper scale of the plot). The solid line gives a fit to the data using Eq. (1), resulting in a tunneling barrier of $0.15 \pm 0.04 \mathrm{eV}$, a value typically found for tunneling of Au electrodes in air. ${ }^{17}$ The observation of the tunneling current clearly demonstrates the proper operation of the developed tunneling sensor. From Fig. 3 it is observed that there are slight differences between the two current curves shown (and also between other curves not shown). We suppose that these differences result from establishing slightly different tunneling positions on the electrode upon each approach. Also some jumps can be observed within one curve, indicated by the arrows for the open squares in Fig. 3; likely atomic changes at the tip or electrode are responsible for these jumps. As discussed by Kenny et al. ${ }^{6,7}$ and Liu and Kenny, ${ }^{8}$ tunneling sensors are limited by stability and mechanical noise issues. A detailed discussion of these issues for the presented tunneling sensor is out of the scope of the present letter.

The demonstrated sensor can be applied to NEMSs, for example, for the frequency detection of ultrahigh frequency nanomechanical resonators. Supplying the tip with actuators that allow lateral and vertical motions converts the tunneling sensor in an integrated STM. A first realization of this is shown in Fig. 4; with the possibilities of micromachining technologies a large number of such STMs could be integrated on one single chip.

In summary, we have presented the design and fabrication of a lateral tunneling sensor for NEMSs. The forces between the tip and the electrode involved in such a sensor were evaluated and requirements for its design determined.

The authors acknowledge financial support from the Spanish government through the MINAHE (TIC200204280-C03-02) and MINAHE 2 projects (TEC2005-07996$\mathrm{CO} 2-01)$.

${ }^{1}$ M. L. Roukes, Technical Digest of the 2000 Solid-State Sensors and Actuators Workshop, 2000, pp. 367-76.

${ }^{2}$ Z. J. Davis, G. Abadal, O. Kuhn, O. Hansen, F. Grey, and A. Boisen, J. Vac. Sci. Technol. B 18, 612 (2000).

${ }^{3}$ C. T.-C. Nguyen, Topical Meeting on Silicon Monolithic Integrated Circuits in RF Systems. Digest of Papers, 2001 (unpublished), pp. 23-32.

${ }^{4}$ J. Verd, G. Abadal, M. Villarroya, J. Teva, A. Uranga, X. Borrisé, F. Campabadal, J. Esteve, E. Figueras, F. Pérez-Murano, Z. Davis, E. Forsen, A. Boisen, and N. Barniol, J. Microelectromech. Syst. 14, 508 (2005).

${ }^{5}$ G. Binnig, H. Rohrer, Ch. Gerber, and E. Weibel, Phys. Rev. Lett. 49, 57 (1982).

${ }^{6}$ T. W. Kenny, S. B. Waltman, J. K. Reynolds, and W. J. Kaiser, Appl. Phys. Lett. 58, 100 (1991).

${ }^{7}$ T. W. Kenny, W. J. Kaiser, S. B. Waltman, and J. K. Reynolds, Appl. Phys. Lett. 59, 1820 (1991).

${ }^{8}$ C.-H. Liu and T. W. Kenny, J. Microelectromech. Syst. 10, 425 (2001). ${ }^{9}$ G. Abadal, Z. J. Davis, B. Helbo, X. Borrisé, R. Ruiz, A. Boisen, F. Campabadal, J. Esteve, E. Figueras, F. Pérez-Murano, and N. Barniol, Nanotechnology 12, 100 (2001).

${ }^{10}$ L. Olsson, N. Lin, V. Yakimov, and R. Erlandsson, J. Appl. Phys. 84, 4060 (1998).

${ }^{11} \mathrm{~S}$. Timoshenko and J. Goodier, Theory of Elasticity (McGraw-Hill, New York, 1970).

${ }^{12} \mathrm{~J}$. N. Israelachvili, Intermolecular and Surface Forces (Academic, London, 1992).

${ }^{13}$ L. Zitzler, S. Herminghaus, and F. Mugele, Phys. Rev. B 66, 155436 (2002).

${ }^{14}$ J. Colchero, A. Storch, M. La, J. Gómez Herrero, and A. M. Baró, Langmuir 14, 2230 (1998).

${ }^{15}$ The restoring forces are calculated based on an initial tip-electrode gap of $1 \mu \mathrm{m}$.

${ }^{16} \mathrm{~A}$ larger width of the beam would result in a more rigid structure allowing us to achieve an even more stable situation; however, the required actuator voltage for the tip approach would get considerably larger, which makes operation less convenient.

${ }^{17}$ M. Binggeli, D. Carnal, R. Nyffenegger, H. Siegenthaler, R. Christoph, and H. Rohrer, J. Vac. Sci. Technol. B 9, 1985 (1991). 